\title{
Influência infantil nas compras de alimentos ultraprocessados: interferência do estado nutricional ${ }^{\#}$ Children's influence over family food purchases of ultra processed foods: interference of nutritional status
}

\author{
Tailane Scapin* \\ Caroline Camila Moreira* \\ Giovanna Medeiros Rataichesck Fiates*
}

\begin{abstract}
Resumo
A criança atua como consumidora influenciadora, direcionando as escolhas dos pais na compra de alimentos em benefício próprio. Entre os alimentos influenciados estão os ultraprocessados, os quais apresentam alta densidade energética, teores elevados de gordura, açúcar ou sal e escassez de fibras. O aumento no consumo desses alimentos, aliado ao declínio do nível de atividade física, explicam, em partes, as crescentes prevalências de sobrepeso e obesidade infantil, observadas nas últimas décadas. O objetivo deste estudo foi então, investigar a influência de crianças nas compras familiares de alimentos ultraprocessados relacionando com o estado nutricional infantil. Estudo transversal, de abordagem quanti-qualitativa, foi realizado com 187 pais de escolares de sete a dez anos matriculados na rede pública de ensino de Florianópolis/SC, no ano de 2011. Foi enviado questionário autoaplicável aos pais e realizada antropometria com os escolares para avaliação do estado nutricional. Praticamente um terço (32,6\%) dos escolares apresentou excesso de peso. Dos 187 pais entrevistados, 181 relataram comprar alimentos a pedido dos filhos, sendo que pais de escolares com excesso de peso o faziam com maior frequência (diferença não significante). Os pais citaram 446 alimentos como sendo os mais pedidos pelos filhos, $84,3 \%$ dos quais foram classificados como ultraprocessados. Os pais de escolares com excesso de peso relataram que seus filhos pediam $23 \%$ mais alimentos ultraprocessados do que os pais daqueles sem excesso de peso. O resultado não foi significante, indicando apenas uma tendência, mas sugerindo que os pais têm dificuldade em resistir aos pedidos dos filhos. Ações que informem e orientem os pais quanto à influência dos filhos nas compras de alimentos seriam importantes para instrumentalizá-los no enfrentamento da situação.
\end{abstract}

Palavras-chave: Criança. Comportamento Alimentar. Alimentos Ultraprocessados. Sobrepeso. Obesidade.

\begin{abstract}
The difference was not statistically significant, but indicates a tendency, suggesting that parents are finding it difficult to refuse their children's requests. Actions to inform and guide parents on the influence of children on their food shopping would be valuable to instrument them on how to deal with the situation. Children act as a person who influences consumers directing their parents' choices towards the purchase of foods for their own intake. Among the most requested foods are the ultra processed ones, with high energy density, high fat, sugar and fat content, and low fiber content. Increased ingestion of such foods, together with low levels of physical activity, explains, in part the growing of the prevalences of child overweight and obesity observed in the last decades. The study's objective was to investigate de influence of children over their parents' food shopping, and relate this to their nutritional status. The transversal quali-quantitative study was carried out among 187 parents conducted from 7 to 10 year old students enrolled in public schools of Florianópolis / SC, in 2001. Surveys were sent to parents and anthropometric measures were obtained from children to evaluate nutritional status. Results showed that nearly one third (32.6\%) of the students were overweight. From the 187 parents, 181 referred buying foods at their children's request, and those whose children were overweight did it more frequently (result not significant). Parents mentioned 446 foods as the most requested by their children. $84.3 \%$ of which were classified as ultra processed. Parents of students with excessive weight reported that their children asked $23 \%$ more ultra processed foods than those parents whose children were not overweight. The difference was not statistically significant, but indicates a tendency, suggesting that parents are finding it difficult to refuse their children's requests. Actions to inform and guide parents on the influence of children on their food shopping would be valuable to instrument them on how to deal with the situation.
\end{abstract}

Keywords: Child. Eating Behavior. Ultra Processed Foods. Overweight. Obesity.

DOI: 10.15343/0104-7809.20153903345353

\# Pesquisa financiada pelo Edital Universal CNPq 2009 (processo 476397/2009).

* Universidade Federal de Santa Catarina, Florianópolis-SC, Brasil. E-mail: giovanna.fiates@ufsc.br

Os autores declaram não haver conflitos de interesse. 


\section{INTRODUÇÃO}

Na sua primeira versão, o Guia Alimentar para a População Brasileira (GAPB) baseava suas recomendações nos conceitos de grupos alimentares e porções, de acordo com a pirâmide alimentar ${ }^{1}$. Tal abordagem levava em consideração alimentos e nutrientes, mas não o processamento, de uso abundante pela indústria alimentar, aplicado aos alimentos atualmente ${ }^{2}$. Em sua nova edição, o GAPB adotou o conceito de grau de processamento para classificar os alimentos, baseado nos estudos do grupo de Monteiro et al. ${ }^{3,4}$. De acordo com a extensão e o propósito do seu processamento, os alimentos são então classificados como in natura ou minimamente processados, como ingredientes culinários (óleos, gorduras, açúcar e sal), como processados, ou como ultraprocessados ${ }^{4}$.

Os alimentos ultraprocessados resultam do processamento de diversos gêneros alimentícios por técnicas variadas, o que os leva a apresentar alta densidade energética, teores elevados de gordura (principalmente saturadas e trans), açúcares e/ou sal, bem como escassez de fibras. Tais alimentos são prontos para o consumo ou prontos para aquecer com pouca ou nenhuma preparação, além de serem altamente palatáveis ${ }^{3,4}$.

Dados sobre o consumo alimentar de 40 mil crianças brasileiras de cinco a dez anos, oriundos do Sistema de Vigilância Alimentar e Nutricional (SISVAN), indicam consumo regular de alimentos ultraprocessados como batata frita, de pacote ou salgadinho frito; hambúrguer e embutidos; biscoitos salgados e salgadinhos de pacote; biscoitos doces e recheados, doces, balas e chocolates; além de refrigerantes ${ }^{5}$.

Estudos também demonstram que alimentos ultraprocessados são os mais consumidos no lanche por escolares brasileiros ${ }^{6,7,8}$, e que o consumo tem sido favorecido pela sua produção abundante, a um custo relativamente baixo $^{9,10,11 .}$

O consumo frequente de alimentos com características de ultraprocessados e insuficiente de leite, suco natural, cereais, vegetais, frutas, carnes e peixes ${ }^{12,13,14}$ tem sido associado com o aumento da incidência de doenças crônicas não transmissíveis e com as crescentes prevalências de sobrepeso e obesidade ${ }^{15,16}$. Na população infantil brasileira com idade entre seis e nove anos, há uma prevalência de $32 \%$ de sobrepeso e $14 \%$ de obesidade, que somados chegam a quase metade da população dessa faixa etária ${ }^{17}$.

Estudos relatam que crianças influenciam as compras familiares, direcionando as escolhas dos pais, direta ou indiretamente, para compra de itens em benefício próprio ${ }^{18,19}$. Dentre os itens cuja compra mais é influenciada por crianças estão os alimentos ${ }^{20}$, principalmente doces, bolachas, chocolates, salgadinhos de pacote, sorvete, pipoca e bebidas açucaradas ${ }^{21-26}$.

Considerando o exposto, o presente estudo objetivou investigar a relação entre o estado nutricional e a influência infantil sobre as compras familiares de alimentos, caracterizados como ultraprocessados.

\section{MÉTODO}

Estudo transversal de abordagem quali-quantitativa, do tipo exploratório e descritivo. A pesquisa foi desenvolvida em 2011 no município de Florianópolis, Santa Catarina, Brasil, envolvendo os responsáveis pelas compras de alimentos para a família de escolares regularmente matriculados em na rede pública municipal de ensino. Tratou-se de um subprojeto do "Estudo intergerações familiares: hábitos alimentares na interface entre o estado nutricional e o comportamento consumidor", contemplado no Edital Universal CNPq 2009 (476397/2009).

Todas as escolas desdobradas do município de Florianópolis/SC $(n=10)$ foram autorizadas pela Secretaria Municipal de Educação a participar do estudo. Houve recusa de uma escola, por alegação de já existirem outras pesquisas sendo desenvolvidas no local. Assim, a amostra final foi composta por nove escolas desdobradas, distribuídas geograficamente por todo o município.

A seleção dos pais responsáveis pelas compras de alimentos para a família se deu por meio dos filhos matriculados nas escolas, de forma censitária. Em 2011, 489 alunos com idades entre sete e dez anos estavam regularmente 
matriculados do primeiro ao quinto ano, e todos levaram para casa convites para os pais. Tais crianças tinham em média 9,2 anos $( \pm 1,01)$, $52,4 \%$ eram do sexo masculino e $56,2 \%$ estudavam no período matutino.

A coleta de dados foi realizada entre setembro e outubro de 2011, mediante o envio de questionários encaminhados aos pais pela agenda escolar da criança, de modo a aumentar a taxa de retorno. Por meio do instrumento de coleta de dados, foram obtidos dados de prevalência de relatos do comportamento de compra de alimentos, bem como de variáveis demográficas e socioeconômicas.

O instrumento utilizado foi um questionário autoaplicável, com perguntas abertas e de múltipla escolha, dividido em duas seções. A primeira sobre dados demográficos e socioeconômicos e a segunda sobre comportamento de compra de alimentos, adaptado de Turner, Kelly e Mckenna ${ }^{27}$. O questionário continha outras variáveis não exploradas no presente artigo.

As variáveis demográficas e socioeconômicas coletadas foram sexo (masculino / feminino), idade (20-29 anos / 30-39 anos / 40-49 anos / 50 anos ou mais), estado civil (solteiro / casado ou com companheiro / separado ou divorciado / viúvo), número de filhos (um / dois ou três / quatro ou mais), escolaridade do principal assalariado da família (0-8 anos / 9-11 anos / 12 anos ou mais) e renda familiar mensal (R\$: $\leq 1020,00 /$ R\$: 1021,00-2040,00 / R\$: $\geq 2041,00$ ou mais), categorizada com base no salário mínimo de 2010 (um salário mínimo equivalia R \$ 510,00), ano em que o instrumento de coleta de dados foi elaborado.

Os dados antropométricos das crianças foram mensurados com balança eletrônica digital portátil, com capacidade para 150 quilos (Kg) e sensibilidade de 50 gramas (g) e fita inelástica e esquadro, com leitura em intervalos de 0,5 centímetros. O estado nutricional das crianças foi classificado com base nos pontos de corte específicos para idade e sexo de acordo com o Índice de Massa Corporal (IMC), segundo padrões da Organização Mundial da Saúde ${ }^{28}$.
A frequência da realização de compras influenciadas pelos filhos foi investigada a partir das respostas à questão: "Você compra algum alimento por causa de pedidos de seu filho?". As opções eram 'sempre', 'às vezes', 'raramente' e 'nunca'. Quanto à caracterização dos alimentos pedidos pelos filhos, os dados foram coletados através da seguinte pergunta aberta: "Se sim, quais alimentos seu filho pede para você comprar?". Os respondentes poderiam citar até três tipos de alimentos.

Os alimentos citados pelos pais como mais pedidos pelos filhos foram categorizados segundo grau de processamento em minimamente processados, ingredientes culinários, processados e ultraprocessados ${ }^{4}$. Alguns alimentos $(n=6)$ não puderam ser classificados pelo critério estabelecido, pois não foi possível constatar o grau de processamento do alimento (ex: suco, quando não havia especificação se era natural ou artificial).

As análises estatísticas foram realizadas por meio do programa STATA Statistical Software Package (versão 11.0, Stata Corp., College Station, TX, USA). No intuito de caracterizar a amostra, os dados foram apresentados inicialmente por meio de uma análise descritiva realizada a partir do número absoluto e da frequência. Posteriormente, para verificar as associações entre as variáveis dependentes e independentes, foram realizadas análises bivariadas, por meio do teste Qui-Quadrado: frequência de compras influenciadas x prevalência de excesso de peso nos escolares. Em todas as análises foi considerado o valor de 5\% como nível de significância estatística $(p<0,05)$.

Foram cumpridos todos os requisitos para pesquisa com seres humanos, previstos pelo Parecer de Aprovação do Comitê de Ética em Pesquisa com Seres Humanos da Universidade Federal de Santa Catarina (1140/10) e pela Secretaria Municipal de Educação de Florianópolis, SC (GEPE 060/2010-004/2011), conforme Resolução 196/96 do Conselho Nacional de Saúde. Os documentos encontram-se arquivados no Departamento de Nutrição da Universidade Federal de Santa Catarina. 


\section{RESULTADOS}

Dos 489 indivíduos convidados a participar, 216 retornaram o questionário preenchido (taxa de resposta de 44,2\%). Foram excluídos das análises 29 indivíduos que não preenchiam os critérios de inclusão, totalizando uma amostra composta por 187 indivíduos (mãe ou pai) responsáveis pelas compras familiares de alimentos.

A Tabela 1 apresenta características demográficas e socioeconômicas dos participantes. A amostra foi constituida predominantemente pelo sexo feminino (88,3\%), com média de idade de 35,4 anos ( \pm 7,2 DP), sendo que metade da amostra encontrava-se na faixa etária de 30 a 39 anos (50,6\%). Aproximadamente 73\% eram casados ou tinham companheiro e mais da metade da amostra tinha dois ou três filhos (53,8\%). A faixa de escolaridade do principal assalariado da família que obteve maior predomínio foi a de 12 ou mais anos de estudo (51,6\%). Quanto à renda familiar mensal, o maior percentual de respondentes (45\%) concentrou-se na faixa de dois a quatro salários mínimos, entre R\$ 1021,00 e R\$2040,00.

A maioria dos pais possuía filhos do sexo feminino (58,3\%, $n=109)$, com média de idade de 8,6 anos $( \pm 1)$. A média de peso e altura das crianças foi de $32,7 \mathrm{~kg}( \pm 7,6 \mathrm{DP})$ e $135,4 \mathrm{~cm}$ $( \pm 7,6 \mathrm{DP})$, respectivamente.

Quanto ao estado nutricional dos escolares $(n=187)$, a maioria encontrava-se eutrófica $(65,8 \%)$. Aproximadamente $1 / 3$ (32,6\%) dos escolares apresentaram excesso de peso, dos quais 20,3\% sobrepeso e 12,3\% obesidade. Menos de $2 \%$ dos escolares apresentaram magreza.

Um total de 181 pais $(96,8 \%)$ relatou comprar alimentos a pedido dos filhos, dos quais $79 \%(n=143)$ referiram que isso ocorria sempre ou às vezes, e apenas $21 \%(n=38)$ mencionaram que ocorria raramente. Pais dos escolares com excesso de peso $(n=61)$ compravam alimentos solicitados pelos filhos com maior frequência (sempre e às vezes), do que os pais dos que não apresentavam excesso de peso $(n=126)$. A diferença, no entanto, não foi estatisticamente significante $(p=0,229)$ (Figura 1).
Tabela 1. Variáveis demográficas e socioeconômicas de pais responsáveis pelas compras familiares de alimentos. Florianópolis/SC, 2011. (n=187)

\begin{tabular}{|c|c|c|}
\hline Variável & $\mathbf{N}$ & $\%$ \\
\hline \multicolumn{3}{|l|}{$\operatorname{Sexo}^{* *}$} \\
\hline Masculino & 20 & 11,7 \\
\hline Feminino & 151 & 88,3 \\
\hline \multicolumn{3}{|l|}{ Idade ${ }^{* *}$} \\
\hline 20-29 anos & 39 & 21,9 \\
\hline 30-39 anos & 86 & 50,6 \\
\hline 40-49 anos & 37 & 21,8 \\
\hline 50 anos ou mais & 8 & 4,7 \\
\hline \multicolumn{3}{|l|}{ Estado civil** } \\
\hline Solteiro & 25 & 14,7 \\
\hline Casado ou com companheiro & 124 & 72,9 \\
\hline Separado ou divorciado & 21 & 12,4 \\
\hline Viúvo & 0 & 0,0 \\
\hline \multicolumn{3}{|l|}{ Número de filhos* } \\
\hline Um & 62 & 34,1 \\
\hline Dois ou três & 98 & 53,8 \\
\hline Quatro ou mais & 22 & 12,1 \\
\hline \multicolumn{3}{|c|}{ Escolaridade do principal assalariado da família*** } \\
\hline $0-8$ anos & 30 & 18,9 \\
\hline 9-11 anos & 47 & 29,6 \\
\hline 12 anos ou mais & 82 & 51,6 \\
\hline \multicolumn{3}{|l|}{ Renda familiar mensal (em reais)** } \\
\hline 1020 ou menos & 62 & 36,7 \\
\hline 1021 a 2040 & 76 & 45,0 \\
\hline 2041 ou mais & 31 & 18,3 \\
\hline
\end{tabular}

* Até $5 \%$ dos dados ignorados

** Até $10 \%$ dos dados ignorados

*** Até $15 \%$ dos dados ignorados

Além do questionamento quanto à frequência de compras, o instrumento de coleta solicitava que fossem mencionados até três exemplos de alimentos cuja compra era influenciada pelos filhos, resultando em 446 citações de alimentos. Os mais frequentemente mencionados foram: bolacha com e sem recheio $(n=83,18,4 \%)$, salgadinho de pacote $(n=65,14,4 \%)$, chocolate $(n=60,13,3 \%)$, frutas $(n=52,11,5 \%)$, iogurte $(n=51,11,3 \%)$ e guloseimas (chicletes e balas) $(n=42,9,3 \%)$. 
Figura 1. Distribuição das compras alimentares influenciadas por escolares da rede pública de ensino de Florianópolis, segundo estado nutricional. Florianópolis/SC, 2011. (n=187)

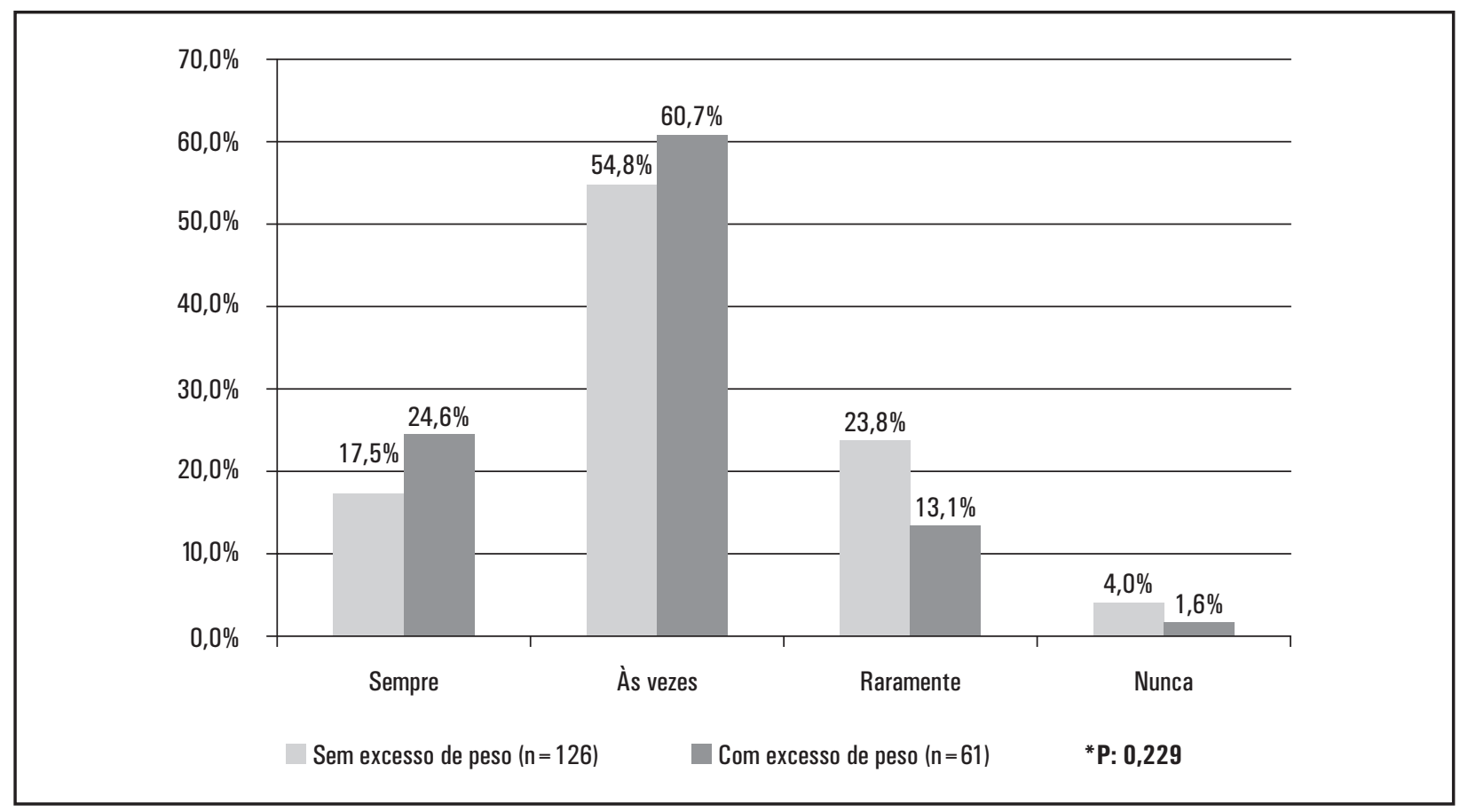

* Teste de Qui-Quadrado

Os 446 alimentos citados foram classificados segundo grau de processamento, de acordo com o novo GAPB ${ }^{4}-84,3 \%$ foram considerados ultraprocessados ( $n=381$ ) e 14,4\% como minimamente processados $(n=65)$. Nenhum alimento foi classificado como ingrediente culinário nem como processado.

A fim de investigar a frequência de pedidos de alimentos ultraprocessados pelos escolares, dentro das três possibilidades de resposta dos pais, foram compilados os dados por indivíduo. O maior percentual da amostra (44\%, $\mathrm{n}=83$ ), citou três alimentos ultraprocessados como pedidos pelo filho, seguido de dois alimentos ultraprocessados $(27,81 \%, \mathrm{n}=52)$, um $(14,97 \%, n=28)$ e nenhum alimento ultraprocessado $(12,87 \%, \mathrm{n}=24)$ (Figura 2$)$.

Dos 83 pais que citaram três alimentos ultraprocessados como pedidos pelos filhos, nas três opções de resposta, 41,3\% $(n=52)$ eram pais de escolares sem excesso de peso. Os demais $50,82 \%(n=31)$ eram pais de escolares com excesso de peso, ou seja, um aumento de $23 \%$, apesar da diferença não ser significante $(p=0,218)$.

\section{DISCUSSÃo}

O estudo transversal quali-quantitativo objetivou analisar, em um grupo de pais de escolares da rede pública de ensino de Florianópolis, o tipo de compra influenciada pelos filhos e sua relação com o estado nutricional das crianças.

No presente estudo, os pais de escolares relataram comprar alimentos a pedido dos fiIhos, principalmente ultraprocessados. Os pais de escolares com excesso de peso relataram $23 \%$ mais alimentos ultraprocessados, além de relatarem atender aos pedidos dos filhos com maior frequência.

Entre os respondentes, a maioria era mulheres, a média de idade foi de 35,4 anos e mais da metade respondeu que o principal assalariado da família possuía 12 anos ou mais de estudo, valor muito superior à média de escolaridade no Brasil na mesma época, que era de 7,2 anos ${ }^{29}$. Dois terços dos pais referiram renda familiar maior que dois salários mínimos mensais. Segundo dados da Pesquisa de Orçamentos Familiares (POF - 2008/2009), os brasileiros obtiveram um acréscimo considerável na renda familiar 
na última década, sendo grande parte gasta na aquisição, e consequente disponibilidade para consumo, de alimentos ${ }^{30}$. Esse montante gasto na alimentação pode explicar o fato de $79 \%$ dos entrevistados relatarem comprar alimentos a pedido dos filhos sempre ou às vezes. Estudo que analisou as tendências de compras e disponibilidade familiar de alimentos, nas últimas três décadas no Brasil, demostrou que houve um acréscimo na aquisição de alimentos caracterizados como ultraprocessados, principalmente nas famílias de maior renda ${ }^{3}$.

Figura 2. Frequência de citação de ultraprocessados pelos pais, segundo o estado nutricional dos escolares. Florianópolis/SC, 2011.

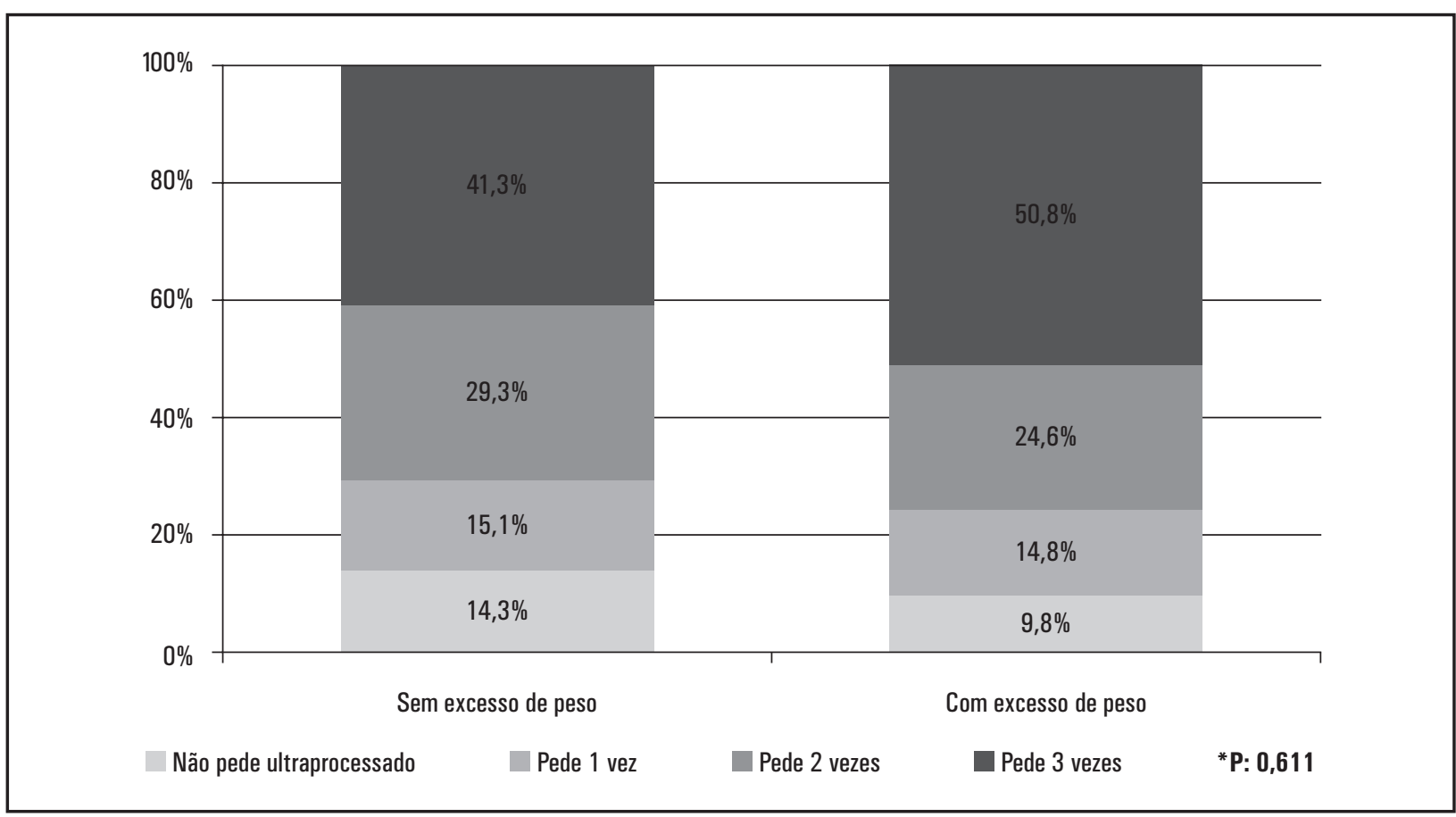

* Teste de Qui-Quadrado

A mesma pesquisa (POF - 2008/2009) demonstrou que houve um aumento importante nas taxas de sobrepeso e obesidade em crianças de seis a nove anos de idade nos últimos dez $\operatorname{anos}^{17}$. Na cidade e no estado em que a presente pesquisa foi realizada, são relatadas prevalências de excesso de peso entre escolares de $21,9 \%$ e $21,4 \%$, respectivamente ${ }^{31,32}$. Na população infantil brasileira, há uma prevalência de $32 \%$ de sobrepeso e $14 \%$ de obesidade, que somados chegam a quase metade da população dessa faixa etária ${ }^{17}$. Tais dados foram semeIhantes ao observado no estudo, onde 32,6\% dos escolares apresentaram excesso de peso (sobrepeso/obesidade).

O fato de pais comprarem com frequência os alimentos pedidos pelos filhos é conhecido e documentado $22,24,33$. No entanto, os estudos identificados não relacionam o estado nutricional das crianças aos tipos de pedidos de compra ou às compras realizadas pelos pais. No presente estudo, os pais dos escolares com excesso de peso relataram comprar com mais frequência os alimentos pedidos pelos filhos, sendo a menção a alimentos ultraprocessados $23 \%$ maior entre os pais de escolares com excesso de peso. Não foram encontrados estudos similares para comparação.

Crianças costumam pedir alimentos de sua preferência aos pais, influenciando sua compra $^{18,21,26}$. Mas, escolhem consumir aqueles alimentos que lhes são servidos mais frequentemente e que estão disponíveis em casa ${ }^{34,35}$. Ou seja, o que os pais disponibilizam no ambiente doméstico pode influenciar e ser influenciado pelas preferências alimentares dos filhos. Se os 
pedidos forem somente por alimentos de baixo valor nutricional, estes estarão disponíveis no ambiente doméstico e poderão ser consumidos não somente pelas crianças, mas por toda a família, influenciando seus hábitos alimentares. Essa situação já foi identificada em um estudo conduzido com pais de estudantes de escola pública e particular de Florianópolis ${ }^{36}$.

No presente estudo, entre os alimentos mencionados pelos pais como solicitados pelos fiIhos, houve predomínio de bolachas, salgadinho de pacote, chocolate e iogurte - alimentos de consumo imediato, resultado similar a estudos anteriores $^{22,33,37}$. Dos alimentos solicitados pelos estudantes, $84,3 \%$ foram classificados como ultraprocessados, ou seja, apresentam densidade energética elevada, altos teores de gordura saturada e/ou trans, açúcar ou sal e escassez de fibras e outros compostos bioativos ${ }^{3}$. Porém, salienta-se que também estavam entre os mais citados as frutas $(11,5 \%)$, o que pode ter gerado a não significância de algumas associações entre pedidos de compra de alimentos ultraprocessados e excesso de peso.

Segundo Louzada et al. ${ }^{38}$, há prejuízos à saúde decorrentes da substituição de refeições tradicionais baseadas em alimentos in natura ou minimamente processados por alimentos ultraprocessados em função do perfil nutricional desses alimentos. Pesquisas com alimentos ultraprocessados demostram consequências de sua ingestão à saúde infanto-juvenil, incluindo o ganho de peso $^{39}$, dislipidemias ${ }^{40}$ e síndrome metabólica ${ }^{41}$.

\section{CONCLUSÃO}

No presente estudo foi verificado que pais de crianças em idade escolar atendiam frequentemente aos pedidos de seus filhos por alimentos ultraprocessados. Apesar de não haver sido observada associação significante com o estado nutricional das crianças, a situação sugere que os pais estão tendo dificuldade de resistir aos pedidos dos filhos por alimentos de baixa qualidade nutricional. Ao classificar os pedidos de alimentos em categorias segundo a extensão, natureza e propósito de processamento, evidenciou-se a necessidade de definir ações que informem e orientem os pais quanto à influência dos filhos nas compras de alimentos, instrumentalizando-os para melhor lidarem com a situação.

\section{REFERÊNCIAS}

1. Brasil. Ministério da Saúde. Guia Alimentar para a População Brasileira: Promovendo a Alimentação Saudável. Brasília. 2006. 210p.

2. Monteiro CA, Levy RB, Claro RM, Castro IRR, Cannon G. A new classification of foods based on the extent and purpose of their processing. Cad Saúde Pública. 2010; 26 (11): 2039-49.

3. Monteiro CA, Levy RB, Claro RM, Castro IRR, Cannon G. Increasing consumption of ultra-processed foods and likely impact on human health: evidence from Brazil. Public Health Nutr. 2011; 14 (1): 5-13.

4. Brasil. Ministério da Saúde. Secretaria de Atenção à Saúde. Departamento de Atenção Básica. Guia Alimentar para a População Brasileira. 2ed. Brasília: Ministério da Saúde, 2014. 156p.

5. Brasil. Ministério da Saúde. Coordenação-Geral da Política de Alimentação e Nutrição. Relatório do Consumo Alimentar por período, fase do ciclo da vida. 2014 [acesso 09 set 2015] Disponível em: http://dabsistemas.saude.gov.br/sistemas/ sisvan/relatorios_publicos/relatorios.php.

6. Pierine DT, Carrascosa APM, Fornazari AC, Watanabe MT, Catalani MCT, Fukuju MM, Silva GN, Maestá N. Composição corporal, atividade física e consumo alimentar de alunos do ensino fundamental e médio. Motriz Rev Educ Fís. 2006 ; 12 (2): 113-24.

7. Gabriel CG, Santos MV, Vasconcelos FAG. Avaliação de um programa para promoção de hábitos alimentares saudáveis em escolares de Florianópolis, Santa Catarina, Brasil. Rev Bras Saúde Mater Infant. 2008; 8 (3): 299-308.

8. Fiates GMR, Müller J, Machado ML, Scapin T, Schweitzer T. Alimentos industrializados consumidos no lanche: composição nutricional e adequação às recomendações do PNAE e ANVISA. Hig Aliment. 2012; 26 (206/207): 190-5.

9. Drewnowski A. Nutrition transition and global dietary trends. Nutrition. 2000; 16 (7/8): 486-7.

10. Nestle M. Food marketing and childhood obesity: A matter of policy. New Engl J Med. 2006; 354 (24): $2527-9$.

11. Monteiro CA, Moubarac JC, Cannon G, Ng SW, Popkin B. Ultra-processed products are becoming dominant in the global food system. Obes Rev. 2013; 14:21-8. 
12. Triches RM, Giugliani ERJ. Obesidade, práticas alimentares e conhecimentos de nutrição em escolares. Rev Saúde Pública. 2005; 39 (4): 541-7.

13. Rinaldi AEM, Pereira AF, Macedo CS, Mota JF, Burini RC. Contribuições das práticas alimentares e inatividade física para o excesso de peso infantil. Rev Paul Pediatr. 2008; 3 (26): 271-7.

14. Costa FFd, Assis MAAd, Leal DB, Campos VC, Kupek E, Conde WL. Mudanças no consumo alimentar e atividade física de escolares de Florianópolis, SC, 2002 - 2007. Rev. Saúde Públ. 2012; 46:117-25.

15. Weaver CM, Dwyer J, Fulgoni VL, King JC, Leveille GA, MacDonald RS, Ordovas J, Schnakenber D. Processed foods: contributions to nutrition. Am J Clin Nutr. 2014; 99 (6): 1525-42.

16. Radominski RB. Aspectos epidemiológicos da obesidade infantil. Rev ABESO. 2011; 49 (49): 1-6.

17. IBGE. Instituto Brasileiro de Geografia e Estatística [Internet]. Pesquisa de Orçamentos Familiares no Brasil 2008/2009:

Antropometria e estado nutricional de crianças, adolescentes e adultos no Brasil. Rio de Janeiro: IBGE. 2010 [acesso 08 set 2015]. Disponível em: http://www.ibge.gov.br/home/estatistica/populacao/condicaodevida/pof/2008_2009_encaa/ default.shtm.

18. Mcneal JU. Kids as consumers: a handbook of marketing to children. New York: Lexington Books; 1992. p.272.

19. Patrick H, Nicklas TA. A review of family and social determinants of children's eating patterns and diet quality. J Am Coll Nutr. 2005; 24(2):83-92.

20. Mcneal JU. Children as consumer of commercial and social products. Washington, DC: Pan American Health Organization; 2000. p.104.

21. Mikkelsen MR, Nørgaard MK. Children's influence on family decision-making in food buying and consumption. Copenhagen Business School. 2006; (3).

22. O'Dougherty M, Story M, Stang J. Observations of parent-child co-shoppers in supermarkets: Children's involvement in food selections, parental yielding, and refusal strategies. J Nutr Educ Behav. 2006; 38: 183-8.

23. Flurry LA. Children's influence in family decision-making: Examining the impact of the changing American family. J Bus Res. 2007; (60): 322-30.

24. Marshall D, O'Donohoe S, Kline S. Families, food, and pester power: Beyond the blame game? J Consum Behav. 2007; 6: 164-81.

25. Fiates GMR, Amboni RDMC, Teixeira E. Television use and food choices of children: Qualitative approach. Appetite. 2008; 50 (1): 12-8.

26. Mazzonetto AC, Fiates GM. Perceptions and choices of Brazilian children as consumers of food products. Appetite. $2014 ; 78: 179-84$.

27. Turner JJ, Kelly J, Mckenna K. Food for thought: parents' perspectives of child influence, Brit Food J. $2006 ; 108$ (3): $181-91$. 28. Onis M, Onyango AW, Borghi E, Siyam A, Nishida C, Siekmann J. Development of a WHO growth reference for school-aged children and adolescents. Bull World Health Org. 2007; 85 (9): 660-7.

29. IBGE. Instituto Brasileiro de Geografia e Estatística [Internet]. Pesquisa de Orçamentos Familiares no Brasil $2008 / 2009$. Censo Demográfico 2010: Características da população e dos domicílios - resultados do universo. Rio de Janeiro: IBGE. 2011 [acesso 08 set 2015]. Disponível em: http://www.ibge.gov.br/home/estatistica/populacao/censo2010/caracteristicas_ da_populacao/default_caracteristicas_da_populacao.shtm

30. IBGE. Instituto Brasileiro de Geografia e Estatística [Internet]. Pesquisa de Orçamentos Familiares no Brasil $2008 / 2009$. Avaliação nutricional da disponibilidade domiciliar de alimentos no Brasil. Rio de Janeiro: IBGE. 2010 [acesso 13 out 2013 ]. Disponível em: http://www.ibge.gov.br/home/estatistica/populacao/condicaodevida/pof/2008_2009_analise_consumo/ 31. Ricardo GD, Caldeira GV, Corso ACT. Prevalência de sobrepeso e obesidade e indicadores de adiposidade central em escolares de Santa Catarina, Brasil. Rev Bras Epidemiol. 2009; 3 (12): 424-35.

32. Bernardo CO, Vasconcelos FAG. Association of parents' nutritional status, and sociodemographic and dietary factors with overweight/obesity in schoolchildren 7 to 14 years old. Cad Saúde Pública. 2012; 2 (28): 291-304.

33. Wilson G, Wood K. The influence of children on parental purchases during supermarket shopping. Int J Consum Stud. 2004; 4 (28): 329-36.

34. Birch LL. Child Feeding Practices and the Etiology of Obesity. J Obes. 2006; 14 (3); 343-4.

35. Geller KS, Dzewaltowski DA. Longitudinal and cross-sectional influences on youth fruit and vegetable consumption. Nutr Rev. 2009; 67 (2): 65-76.

36. Dallazen C. Percepção de pais de escolares sobre o comportamento de seus filhos como influenciadores das compras familiares de alimentos [dissertação]. Florianópolis: Universidade Federal de Santa Catarina, Programa de Pós-Graduação em Nutrição, Departamento de Nutrição; 2012.

37. Nørgaard MK, Bruns K, Christensen PH, Mikkelsen MR. Children's influence on and participation in the family decision process during food buying. Young Consumers. 2007; 8 (3): 197-216.

38. Louzada MLdC, Martins APB, Canella DS, Baraldi LG, Levy RB, Claro RM, Moubarac, J-C, Cannon G, Monteira CA. Ultra-processed foods and the nutritional dietary profile in Brazil. Ver Saúde Públ. 2015; 49:01-11.

39. Canella DS, Levy RB, Martins AP, Claro RM, Moubarac JC, Baraldi LG, Cannon G, Monteiro CA. Ultra-processed food products and obesity in Brazilian households (2008-2009). PLoS ONE. 2014; 9(3):e92752. 
40. Rauber F, Campagnolo PDB, Hoffman DJ, Vitolo MR. Consumption of ultra-processed food products and its effects on children's lipid profiles: A longitudinal study. Nutr Metab Cardiovasc Dis. 2015; 25(1):116-22.

41. Tavares LF, Fonseca SC, Garcia Rosa ML, Yokoo EM. Relationship between ultra-processed foods and metabolic syndrome in adolescents from a Brazilian Family Doctor Program. Public Health Nutr. 2012; 15(1):82-7. 